\title{
Segregation and pursuit waves in activator-inhibitor systems
}

\author{
Vicenç Méndez, ${ }^{1}$ Werner Horsthemke, ${ }^{2}$ Evgeny P. Zemskov, ${ }^{3,4}$ and José Casas-Vázquez ${ }^{1}$ \\ ${ }^{1}$ Grup de Física Estadística, Departament de Física, Facultat de Ciències, Edicifi Cc. Universitat Autònoma de Barcelona, \\ E-08193 Bellaterra (Barcelona), Spain \\ ${ }^{2}$ Department of Chemistry, Southern Methodist University, Dallas, Texas 75275-0314, USA \\ ${ }^{3}$ Computing Centre of the Russian Academy of Sciences, Vavilova 40, 119991 Moscow, Russia \\ ${ }^{4}$ Department of Physics and Astronomy, University of New Mexico, 800 Yale Boulevard, Albuquerque, New Mexico 87131, USA
}

(Received 18 July 2006; revised manuscript received 7 September 2007; published 25 October 2007)

\begin{abstract}
We investigate the effects of cross-diffusion on propagating waves in an activator-inhibitor system. The model consists of a piecewise linear approximation of FitzHugh-Nagumo kinetics and a cross-diffusion term for either the activator or the inhibitor. We obtain exact analytic solutions for traveling fronts and solitary pulses and discuss the corresponding speed diagrams. A detailed comparison with the corresponding RinzelKeller model for the usually studied case of self-diffusion is performed.
\end{abstract}

DOI: $10.1103 /$ PhysRevE.76.046222

PACS number(s): 05.45.-a, 02.50.- r

\section{INTRODUCTION}

Spatiotemporal pattern formation in physical, chemical, biological, ecological, and other systems has been modeled frequently by reaction-diffusion equations [1-5]. Most reaction-diffusion models assume that the diffusion matrix is a diagonal matrix, i.e., the diffusive flux of a given species is only driven by the gradient of that species. Models with offdiagonal terms in the diffusion matrix, representing crossdiffusion, have attracted attention recently. The effect of cross-diffusive terms on pattern formation has been investigated in the context of population dynamics [6]. Particular applications include the spatial segregation of interacting populations $[7,8]$, the asymptotic behavior of epidemic models with pursuit and evasion movements [9], the seed production in forests with two age classes [10], Turing patterns in activator-inhibitor systems [11], predator-prey systems in one dimension [12], and quasisolitons in the FitzHughNagumo [13] system [6]. Moreover, cross-diffusion has found applications in a purely physical setting, such as binary and multicomponent mixtures, concentrated liquid mixtures, and binary Lennard-Jones fluid mixtures (see, e.g., Ref. [14], and references therein). Cross-diffusion has also been investigated in the segregation of magnetically confined fusion plasmas [15]. From a fundamental point of view, we note that cross-diffusion is present in the formalism of nonequilibrium thermodynamics of multicomponent systems [16].

We investigate the effects of cross-diffusion in an activator-inhibitor system. To determine those features in the dynamics that are specifically caused by the cross-diffusion terms, we consider the limiting case that the species do not undergo self-diffusion, i.e., the diagonal terms of the diffusion matrix vanish. This case allows us to elucidate how traveling waves emerge due to a combination of excitable kinetics and the segregation or pursuit movement between the activator and inhibitor species caused by cross-diffusion. The nonlinearity in the local kinetics has the form of a FitzHugh-Nagumo function [13]. To obtain exact analytic solutions for general solitary waves, fronts and pulses, for the systems, we adopt a piecewise linear caricature [17] of the reaction term, namely, the Rinzel-Keller function [18]. The paper is organized as follows. In Sec. II we motivate and introduce the reaction-diffusion equations we study and show that they exhibit traveling waves whose speed has an upper bound. In Sec. III we determine the conditions for the existence of segregation fronts and pulses. We also obtain exact analytical expressions for these waves and highlight the main differences between the case of pure cross-diffusion we study here and the commonly studied case of pure selfdiffusion. We show in Sec. IV that pursuit waves cannot exist, either as fronts or as pulses. In the concluding Sec. V we summarize and highlight our main results.

\section{THE MODEL EQUATIONS}

The evolution equations for a reaction-diffusion system have the form

$$
\frac{\partial n_{i}}{\partial t}=\sum_{j=1}^{m} D_{i j} \frac{\partial^{2} n_{j}}{\partial x^{2}}+F_{i}(n)
$$

where $\mathrm{D}=\left(D_{i j}\right)$ is the diffusion matrix, $F_{i}(n)$ describes the kinetics of species $i, n_{j}$ denotes the field variable of species $j$, $j=1, \ldots, m$, and $n=\left(n_{1}, n_{2}, \ldots, n_{m}\right)$. As mentioned before, most treatments of multivariable reaction-diffusion systems assume that the diffusion matrix $\mathrm{D}$ is diagonal, $D_{i j}=0$ for $i$ $\neq j$, i.e., cross-diffusion is negligible. If the cross-diffusion term is positive, $D_{i j}>0$, then the flux of species $i$ is directed toward decreasing values of the field of species $j$, whereas $D_{i j}<0$ implies that the flux is directed toward increasing values of the field of species $j$.

In the following we consider a two-component system, namely, an activator-inhibitor system. Let species 1 be the activator and species 2 the inhibitor. We adopt the following notation $n_{1}=u$ and $n_{2}=v$. To explore and isolate those features in the dynamics of activator-inhibitor systems that arise specifically from cross-diffusive effects, we consider the limiting case of vanishing self-diffusion $D_{i i}=0$. We set $D_{12}$ $\equiv h_{v}, D_{21} \equiv-h_{u}, F_{1}(u, v)=f(u)-v$, and $F_{2}(u, v)=\varepsilon(u-b v)$. The positive sign of $D_{12}$ implies that the activator segregates from the inhibitor, and $D_{21} \equiv-h_{u}<0$ implies pursuit of the 
activator by the inhibitor. The evolution equations for the activator and inhibitor read

$$
\begin{gathered}
\frac{\partial u}{\partial t}=f(u)-v+h_{v} \frac{\partial^{2} v}{\partial x^{2}}, \\
\frac{\partial v}{\partial t}=\varepsilon(u-b v)-h_{u} \frac{\partial^{2} u}{\partial x^{2}} .
\end{gathered}
$$

We choose the piecewise-linear Rinzel-Keller approximation of the FitzHugh-Nagumo function for the kinetics of the activator, $f(u)=-u+\theta(u-a)$. Here $\theta(u-a)$ is the Heaviside step function. The positive parameter $\varepsilon$ represents the ratio of the time scales, and the parameters $a, b, h_{v}$, and $h_{u}$ are positive and constant.

We study two cases of Eq. (1): (i) a mobile activator segregates from an immobile inhibitor, $h_{v}=1$ and $h_{u}=0$ and (ii) a mobile inhibitor pursues an immobile activator, $h_{v}=0$ and $h_{u}=1$. This simplification allows us to isolate the effects of activator cross-diffusion and the effects of inhibitor crossdiffusion. The contributions of the cross-diffusion of each species to the overall dynamics are harder to untangle for the case where both the activator and inhibitor are mobile, and that case will not be dealt with here. Traveling waves result from the interplay of two effects, namely, interaction between the species and spatial dispersal. If one or both effects are missing, a traveling wave cannot exist. In case (i), where a mobile activator segregates from an immobile inhibitor, we expect that traveling waves exist only if segregation does not occur too fast. Otherwise, no interaction between activator and inhibitor occurs and traveling waves cannot emerge. Consequently, we anticipate the existence of an upper bound for the wave speed, caused by the upper bound on the diffusion speed of the activator. The existence of upper bounds for the propagation speed of traveling waves is characteristic of reaction-diffusion equations of hyperbolic type. Indeed, Eq. (2) can be regarded as a hyperbolic reaction-diffusion (HRD) equation, as we show at the end of this section. In case (ii), where the activator is immobile and the inhibitor moves into regions where the activator is present, the interaction is very successful but no traveling wave can emerge because the movement of the inhibitor tends to concentrate it in a small region of space. We anticipate that in this case no pursuit waves exist, and our analytic results will confirm this conjecture.

We end this section with establishing the connection between the activator-inhibitor system with cross-diffusion (2) and HRD equations [19]. For case (i), $h_{v}=1$ and $h_{u}=0$, taking the temporal derivative of Eq. (2a) and using Eqs. (2a) and $(2 \mathrm{~b})$ to eliminate $v$, we obtain the HRD equation for the activator

$$
\frac{\partial^{2} u}{\partial t^{2}}+\varepsilon b \frac{\partial u}{\partial t}=\varepsilon \frac{\partial^{2} u}{\partial x^{2}}-\varepsilon u+\varepsilon b f(u)+\frac{\partial f(u)}{\partial t} .
$$

From a physical point of view, this result implies that the activator-inhibitor system with cross-diffusion (2) displays traveling waves only under certain conditions for the parameters and that the speeds of traveling fronts must be bounded from above. The same results hold also for case (ii), $h_{v}=0$ and $h_{u}=1$. In this case, the HRD equation for the activator is

$$
\frac{\partial^{2} u}{\partial t^{2}}+\varepsilon b \frac{\partial u}{\partial t}=\frac{\partial^{2} u}{\partial x^{2}}-\varepsilon u+\varepsilon b f(u)+\frac{\partial f(u)}{\partial t} .
$$

The HRD equations for cases (i) and (ii) differ only in the effective diffusion constant in the HRD equations (3) and (4). The diffusion coefficient is $\varepsilon$ in the case of a mobile activator and an immobile inhibitor and 1 in the case of an immobile activator and a mobile inhibitor. The time scale of the immobile species, which drives the mobile species via cross-diffusion, determines the effective diffusion coefficient.

\section{SEGREGATION WAVES}

The system with a mobile activator and an immobile inhibitor

$$
\begin{gathered}
\frac{\partial u}{\partial t}=-u-v+\theta(u-a)+\frac{\partial^{2} v}{\partial x^{2}}, \\
\frac{\partial v}{\partial t}=\varepsilon(u-b v)
\end{gathered}
$$

always has a trivial homogeneous steady state (HSS),

$$
(\bar{u}, \bar{v})=(0,0) .
$$

If the condition

$$
0<a<\frac{b}{1+b}
$$

is fulfilled, which we assume in the following, then a second, nontrivial HSS exists,

$$
(\bar{u}, \bar{v})=\left(\frac{b}{1+b}, \frac{1}{1+b}\right) .
$$

Both states are linearly stable steady states of the homogeneous system, i.e., system (5) without the spatial term.

A traveling front is a heteroclinic connection joining the trivial HSS and the nontrivial HSS, while a traveling pulse (a homoclinic connection) leaves the trivial HSS, rises to values near the nontrivial HSS, and then returns to the trivial HSS. Both trivial and nontrivial HSSs have the same Jacobian matrix, so that, both have the same strength of attraction and traveling fronts can propagate to the right or to the left. We introduce the traveling-frame coordinate $\xi=x-c t$, where $c$ is the propagation speed, and rewrite system (5) as

$$
\begin{gathered}
\frac{d^{2} v}{d \xi^{2}}+c \frac{d u}{d \xi}-u-v+\theta(u-a)=0 \\
c \frac{d v}{d \xi}+\varepsilon u-\varepsilon b v=0 .
\end{gathered}
$$

The system (9) is linear and the solutions are linear combinations of exponential functions

$$
u(\xi)=\sum_{k} A_{k} e^{\lambda_{k} \xi}+\bar{u}, \quad v(\xi)=\sum_{k} B_{k} e^{\lambda_{k} \xi}+\bar{v},
$$

where $A_{k}$ and $B_{k}$ are integration constants 
To evaluate explicitly the solutions (10), we insert them into Eq. (9). Collecting terms, we obtain, omitting the subscript $k$ for notational simplicity

$$
\left(\begin{array}{cc}
c \lambda-1 & \lambda^{2}-1 \\
\varepsilon & c \lambda-\varepsilon b
\end{array}\right)\left(\begin{array}{l}
A \\
B
\end{array}\right)=0 .
$$

The characteristic equation $(c \lambda-1)(c \lambda-\varepsilon b)-\varepsilon\left(\lambda^{2}-1\right)=0$ has the roots

$$
\lambda^{ \pm}=\rho \pm \sqrt{\rho^{2}+\sigma}, \quad \text { where } \rho \equiv-\frac{c}{2} \frac{1+\varepsilon b}{\varepsilon-c^{2}}, \quad \sigma \equiv \varepsilon \frac{1+b}{\varepsilon-c^{2}} .
$$

Equation (11) implies that the integration constants fulfill $B_{i}^{ \pm}=\gamma^{ \pm} A_{i}^{ \pm}$, where $\gamma^{ \pm}=\varepsilon /\left(\varepsilon b-c \lambda^{ \pm}\right)$. If $\varepsilon>c^{2}$, then $\lambda^{+}>0$ and $\lambda^{-}<0$. If $\varepsilon<c^{2}$, both $\lambda^{ \pm}$are positive, and propagating waves cannot exist. Consequently, the condition $|c|<\sqrt{\varepsilon}$ is required to guarantee the existence of traveling waves. Note that this condition is equivalent to the upper bound on the speed of HRD fronts, see Eq. (13) in Ref. [19]. In summary, segregation waves only exist if two conditions are fulfilled:

$$
-\sqrt{\varepsilon}<c<\sqrt{\varepsilon} \text { and } 0<a<\frac{b}{1+b} .
$$

\section{A. Segregation fronts}

Let us first consider the front solutions of Eq. (9). Each front consists of two pieces

$$
\begin{aligned}
& u(\xi)= \begin{cases}u_{1}(\xi)=A^{+} e^{\lambda^{+} \xi}, & \xi \leqslant 0, \\
u_{2}(\xi)=A^{-} e^{\lambda^{-} \xi}+\bar{u}, & \xi \geqslant 0,\end{cases} \\
& v(\xi)= \begin{cases}v_{1}(\xi)=B^{+} e^{\lambda^{+} \xi}, & \xi \leqslant 0, \\
v_{2}(\xi)=B^{-} e^{\lambda^{-} \xi}+\bar{v}, & \xi \geqslant 0 .\end{cases}
\end{aligned}
$$

The front solution must be continuous at $\xi=0$, i.e., $u_{1}(0)$ $=u_{2}(0)=a$ and $v_{1}(0)=v_{2}(0)$. These conditions imply $A^{+}=a$ and $A^{-}=a-\bar{u}$. The relation $B^{ \pm}=\gamma^{ \pm} A^{ \pm}$yields the equation for the front speed, $a\left(\gamma^{+}-\gamma^{-}\right)+\bar{u} \gamma^{-}-\bar{v}=0$. This equation has the trivial solution $c \equiv 0$, i.e., a stationary front. The other solutions may be found from

$$
a=\frac{1}{1+b} \frac{\lambda^{-}}{\varepsilon} \frac{c \lambda^{+}-\varepsilon b}{\lambda^{+}-\lambda^{-}} .
$$

In Fig. 1 we plot the segregation front profiles for the activator and inhibitor, given by Eqs. (14), traveling to the right. Note that requiring continuity of the solution is sufficient to obtain all the integration constants and the speed equation. The derivatives of $u$ and $v$ need not be continuous, and indeed Figure 1 shows clearly that the curves $u=u(\xi)$ and $v=v(\xi)$ display a kink at $\xi=0$.

Equation (15) implies that the sign of the derivative $\partial a / \partial c$ equals the sign of $b^{2} \varepsilon-b-2$. In other words, the slope of the velocity curves $c=c(a)$ does not depend on the value of $a$; they are either monotonically increasing or monotonically decreasing functions for all values of $a$. In contrast, velocity

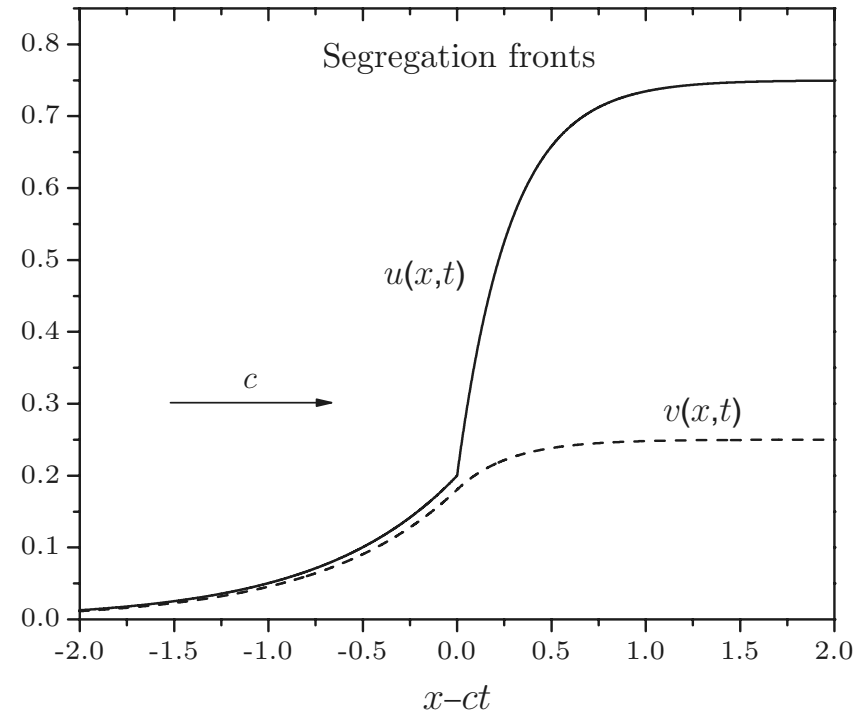

FIG. 1. Plot of segregation front profiles for the activator (solid curve) and the inhibitor (dashed curve), computed from Eqs. (14), for $\varepsilon=0.1, b=3$, and $a=0.2$. Equation (15) yields the front speed $c=0.137$, and fronts propagate to the right.

curves $c=c(a)$ for the self-diffusion case [20] can display two branches for certain conditions. In that case it is necessary to determine which branch corresponds to the branch of stable fronts. In our case $c=c(a)$ is a single-valued function, and it is not necessary to study its stability; if a front exists, it is always stable. Equation (15) and conditions (13) lead to the following cases: (i) If $0<\varepsilon b^{2}<1, c$ decreases monotonically and $c_{\max }=c(a=0)=\varepsilon b$ and $c_{\min }=c(a=\bar{u})=-\varepsilon b$. (ii) If $1<\varepsilon b^{2}<2+b, c$ decreases monotonically. However, fronts only exist if

$$
\frac{\varepsilon b^{2}-1}{(1+b)(1+\varepsilon b)}<a<\frac{1}{1+\varepsilon b} .
$$

(iii) If $\varepsilon b^{2}>2+b, c$ increases monotonically, and again fronts only exist if Eq. (16) is fulfilled. Cases (i) and (ii) are typical behavior for bistable systems. The larger $a$, the higher the threshold, the more difficult it is to excite the front, and the front speed decreases with $a$. However, in case (iii), i.e., $\varepsilon b^{2}>2+b$, the front speed increases monotonically with $a$, in contrast to the self-diffusion case.

Figure 2 shows the speed curves $c(a)$, computed from Eq. (15), for different values of $b$ and $\varepsilon$. We have chosen $b=1$, $b=4$ and $\varepsilon=0.1, \varepsilon=0.2$, as in Ref. [20]. Two similarities exist between the self-diffusion and cross-diffusion cases. First, the speed curve is not defined for all values due to the conditions (13). Second, there exist values of $a$ where the front speed equals zero,

$$
a_{c=0}=\frac{b}{2(1+b)} .
$$

Note that $a_{c=0}$ does not depend on $\varepsilon$, as also illustrated in Fig. 2. The main difference between the self-diffusion and cross-diffusion cases lies in the shape of the speed curves. For the cross-diffusion case, the sign of the derivative $\partial a / \partial c$ 


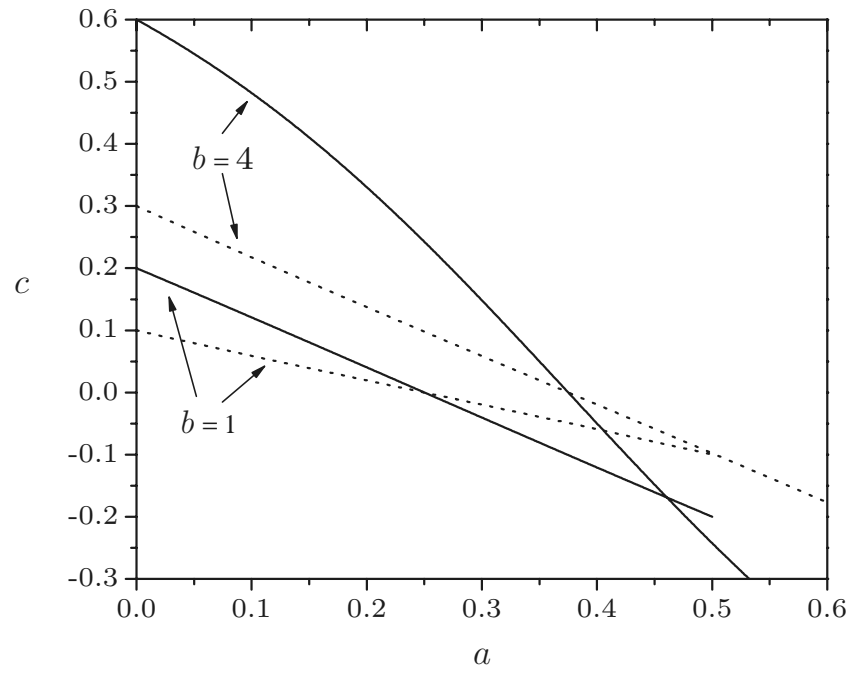

FIG. 2. Plot of the propagation speed $c=c(a, b)$ of segregation fronts as a function of $a$ for various positive values of $b$ and for two ratios of the time scales $\varepsilon$, namely, $\varepsilon=0.1$ (dashed line) and $\varepsilon$ $=0.2$ (solid line). The speed curves are obtained from Eq. (15).

does not depend on $a$ and the speed curves are single-valued functions of $a$, as explained above. In contrast, the speed curves have two branches forming a knee for $b=1$ in the self-diffusion case (see Fig. 5 in Ref. [20]).

\section{B. Segregation pulses}

Let us now consider pulse solutions of Eq. (9). Pulses consist of three pieces

$$
\begin{gathered}
u(\xi)= \begin{cases}u_{1}(\xi)=A_{1}^{+} e^{\lambda^{+} \xi}, & \xi \leqslant 0, \\
u_{2}(\xi)=A_{2}^{+} e^{\lambda^{+} \xi}+A_{2}^{-} e^{\lambda^{-} \xi}+\bar{u}, & 0 \leqslant \xi \leqslant \xi_{0}^{*}, \\
u_{3}(\xi)=A_{3}^{-} e^{\lambda^{-} \xi}, & \xi \geqslant \xi_{0}^{*},\end{cases} \\
v(\xi)= \begin{cases}v_{1}(\xi)=B_{1}^{+} e^{\lambda^{+} \xi}, & \xi \leqslant 0, \\
v_{2}(\xi)=B_{2}^{+} e^{\lambda^{+} \xi}+B_{2}^{-} e^{\lambda^{-} \xi}+\bar{v}, & 0 \leqslant \xi \leqslant \xi_{0}^{*}, \\
v_{3}(\xi)=B_{3}^{-} e^{\lambda^{-} \xi}, & \xi \geqslant \xi_{0}^{*} .\end{cases}
\end{gathered}
$$

Requiring continuity of the solutions and the first derivatives, we obtain

$$
\begin{gathered}
A_{1}^{+}=a, \\
A_{2}^{+}+A_{2}^{-}+\bar{u}=a, \\
\gamma^{+} A_{1}^{+}=\gamma^{+} A_{2}^{+}+\gamma^{-} A_{2}^{-}+\bar{v}, \\
A_{2}^{+} e^{\lambda^{+} \xi_{0}^{*}}+A_{2}^{-} e^{\lambda^{-} \xi_{0}^{*}}+\bar{u}=A_{3}^{-} e^{\lambda^{-} \xi_{0}^{*}}, \\
\gamma^{+} A_{2}^{+} e^{\lambda^{+} \xi_{0}^{*}}+\gamma^{-} A_{2}^{-} e^{\lambda^{-} \xi_{0}^{*}}+\bar{v}=\gamma^{-} A_{3}^{-} e^{\lambda^{-} \xi_{0}^{*}},
\end{gathered}
$$

$$
A_{3}^{-} e^{\lambda^{-} \xi_{0}^{*}}=a .
$$

The procedure for determining the integration constants is the same as in Ref. [21], and we omit it here for the sake of conciseness. The results read

$$
\begin{gathered}
A_{1}^{+}=a \\
A_{2}^{+}=a+\frac{\gamma^{-} \bar{u}-\bar{v}}{\gamma^{+}-\gamma^{-}}, \\
A_{2}^{-}=-\frac{\gamma^{+} \bar{u}-\bar{v}}{\gamma^{+}-\gamma^{-}}, \\
A_{3}^{-}=\frac{a}{1-a\left(\gamma^{+}-\gamma^{-}\right) /\left(\gamma^{+} \bar{u}-\bar{v}\right)} .
\end{gathered}
$$

Equations (19) yield also the coordinate of the second matching point

$$
\xi_{0}^{*}=\frac{1}{\lambda^{-}} \ln \left(1-a \frac{\gamma^{+}-\gamma^{-}}{\gamma^{+} \bar{u}-\bar{v}}\right)
$$

and the pulse speed equation

$$
\lambda^{+} \ln \left(1-a \frac{\gamma^{+}-\gamma^{-}}{\gamma^{+} \bar{u}-\bar{v}}\right)+\lambda^{-} \ln \left(1+a \frac{\gamma^{+}-\gamma^{-}}{\gamma^{-} \bar{u}-\bar{v}}\right)=0
$$

The construction (18) holds only if $\lambda^{+}>0$ and $\lambda^{-}<0$, i.e., if $\varepsilon>c^{2}$. A second condition for the existence of pulses is provided by Eq. (21). Since $\xi_{0}^{*}$ is positive, it is necessary that

$$
a<\frac{\lambda^{+}\left(\varepsilon b-c \lambda^{-}\right)}{(1+b) \varepsilon\left(\lambda^{+}-\lambda^{-}\right)} .
$$

Equation (22) yields a third condition; namely,

$$
-\varepsilon b<c<\varepsilon b \quad \text { and } \quad a<\frac{\lambda^{-}\left(-\varepsilon b+c \lambda^{+}\right)}{(1+b) \varepsilon\left(\lambda^{+}-\lambda^{-}\right)}
$$

must be satisfied simultaneously. When $c=0$, Eq. (22) transforms into an identity, i.e., a trivial solution is the stationary pulse, which exists for any value of $a, \varepsilon$ and $b \neq 0$. This characteristic feature of the cross-diffusion case does not occur for the self-diffusion case. Conditions (23) and (24) restrict the parameter space $(a, c)$ in such a way that propagating pulses can exist only if $\varepsilon b^{2}>1$, in contrast to the selfdiffusion case. Also $c=c(a)$ must be a double-valued function. For fixed $a$, the pulse speed takes a positive and a negative value with the same absolute value. In other words, the system displays two counterpropagating pulses with the same absolute speed. Further, pulses (same as fronts) have a speed bounded by $\sqrt{\varepsilon}$, but fronts always travel faster than pulses. The above conditions can be summarized as a condition on $a$ such that pulses exist if $a_{\min }<a<a_{\max }$, where $a_{\min }$ and $a_{\max }$ are certain functions of $b$ and $\varepsilon$. In Fig. 3 we plot segregation pulse profiles computed from Eqs. (18). In Fig. 4 , we give an example of two speed curves $c=c(a)$ for $b$ $=4$ and $b=8$ with $\varepsilon=0.5$. Each diagram shows a pitchfork bifurcation, which is shifted when $b$ and $\varepsilon$ change. Since we obtain up to three possible values for the speed at a given 


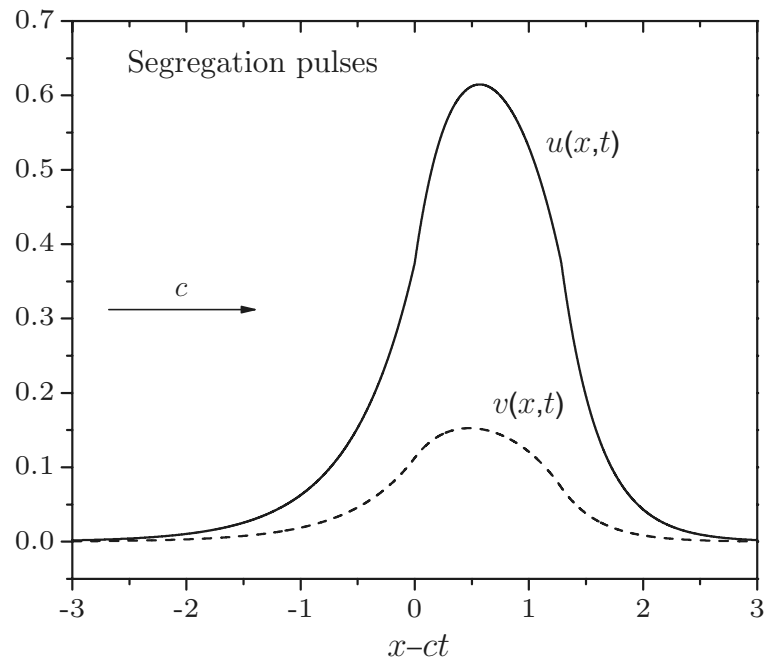

FIG. 3. Plot of segregation pulse profiles for the activator (solid curve) and the inhibitor (dashed curve), computed from Eq. (18), for $\varepsilon=0.5, b=4$, and $a=0.375$. Equation (22) yields the pulse speed $c=0.189$, and pulses propagate to the right.

value of $a$, the pulse speed that is actually selected needs to be determined by a stability analysis. This turns out to be a rather arduous task and will be reported elsewhere.

\section{PURSUIT WAVES}

We now consider case (ii), where the activator is immobile and the inhibitor experiences cross-diffusion,

$$
\begin{gathered}
\frac{\partial u}{\partial t}=-u-v+\theta(u-a), \\
\frac{\partial v}{\partial t}=\varepsilon(u-b v)-\frac{\partial^{2} u}{\partial x^{2}} .
\end{gathered}
$$

For this case Eq. (11) reads

$$
\left(\begin{array}{cc}
c \lambda-1 & -1 \\
-\left(\lambda^{2}-\varepsilon\right) & c \lambda-\varepsilon b
\end{array}\right)\left(\begin{array}{l}
A \\
B
\end{array}\right)=0
$$

so that $\rho$ and $\sigma$ in the roots $\lambda^{ \pm}=\rho \pm \sqrt{\rho^{2}+\sigma}$ of the characteristic equation are

$$
\rho \equiv-\frac{c}{2} \frac{1+\varepsilon b}{1-c^{2}}, \quad \sigma \equiv \varepsilon \frac{1+b}{1-c^{2}} .
$$

Consequently, waves only exist if $|c|<1$. Front solutions, pulse solutions, and the matching equations remain formally the same as for case (i), but $\rho$ and $\sigma$ are now given by Eq. (27). Therefore, the front speed equation takes the same form as above, i.e., $a\left(\gamma^{+}-\gamma^{-}\right)+\bar{u} \gamma^{-}-\bar{v}=0$, where now $\gamma^{ \pm}=c \lambda^{ \pm}$ -1 , and we obtain

$$
a=\frac{1-b\left(c \lambda^{-}-1\right)}{c(1+b)\left(\lambda^{+}-\lambda^{-}\right)} .
$$

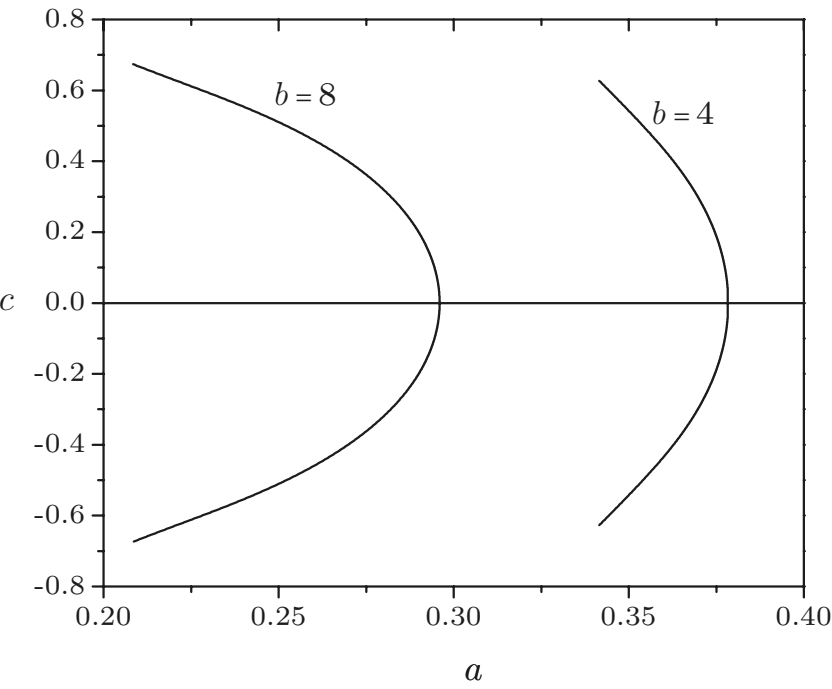

FIG. 4. Plot of the propagation speed $c=c(a, b)$ of a pulse as a function of $a$ for various positive values of $b$ and for a ratio of the time scales of $\varepsilon=0.5$. The speed curves are obtained from Eq. (22).

The interval of $a$ is limited by the requirement that two HSSs exist and by the conditions (13), which now read

$$
-1<c<1 \quad \text { and } \quad 0<a<\frac{b}{1+b} .
$$

Equation (28) implies that the front speed is positive only for $a<0$, which is incompatible with Eq. (29). The front speed is negative for positive values of $a$, if $a$ is larger than $\bar{u}$, which is also incompatible with Eq. (29). We conclude therefore that pursuit waves do not exist, as anticipated.

\section{CONCLUSIONS}

We have considered an activator-inhibitor system with cross-diffusion in one of the components in order to study segregation and pursuit waves. When the activator is immobile and the inhibitor segregates, propagating fronts and pulses appear for appropriate conditions on the parameters. We have shown that our model is equivalent to a HRD equation, which implies that an immobile component decreases the front or pulse speed, as is the case for HRD waves. In particular, we have found that the upper bound for the front speed is $\sqrt{\varepsilon}$, where $\varepsilon$ is the ratio of the activator and inhibitor time scales. Second, we have obtained exact, analytic expressions for the front and pulse solutions and discussed the similarities and differences between our speed diagrams for the case of cross-diffusion and those of the classical RinzelKeller model for the commonly studied case of selfdiffusion. The existence conditions for fronts and pulses have been analyzed in detail. In particular, we have shown that segregation fronts are unique, which is not true in the selfdiffusion case. The front speed increases or decreases as a function of the threshold parameter $a$, in contrast to the selfdiffusion case where the selected front speed always decreases as $a$ increases. For segregation pulses, the speed curve bifurcates, and two counterpropagating pulses emerge that coexist with the trivial (stationary) pulse solution. In the 
self-diffusion case, in contrast, only pulses with positive speeds exist. In addition we have shown that segregation fronts travel always faster than segregation pulses. The stability analysis of these solutions will be dealt with elsewhere. As expected, for pursuit waves, where the activator is immobile and the inhibitor moves toward it, no traveling waves, neither fronts nor pulses, exist.

The piecewise linear approximation we used can be extended to systems with more complicated nonlinear reaction terms, leading to a generalization to multistable cases [21]. Our work has implications for population models with Allee effects as well as for a wide variety of physical, chemical, and biological systems with nonlinear kinetics and crossdiffusion interaction between fields.

\section{ACKNOWLEDGMENTS}

This work was inspired by M. A. Tsyganov's seminar presentation at the Biophysics Department, Faculty of Biology, Moscow State University. E.P.Z. thanks him for introducing us to taxis waves and V. N. Biktashev for reading the manuscript and sending Ref. [6]. V.M. and J.C.-V. acknowledge the Grants Nos. FIS 2006-12296-C02-01 and SGR 200500087.
[1] M. C. Cross and P. C. Hohenberg, Rev. Mod. Phys. 65, 851 (1993).

[2] G. Nicolis and I. Prigogine, Self-Organization in Nonequilibrium Systems (Wiley, New York, 1977).

[3] J. D. Murray, Mathematical Biology II: Spatial Models and Biomedical Applications (Springer, New York, 2003).

[4] A. Okubo and S. A. Levin, Diffusion and Ecological Problems (Springer, New York, 2001).

[5] D. Walgraef, Spatio-Temporal Pattern Formation (Springer, New York, 1996).

[6] V. N. Biktashev and M. A. Tsyganov, Proc. R. Soc. London, Ser. A 461, 3711 (2005)

[7] M. E. Gurtin, Q. Appl. Math. 32, 1 (1974).

[8] N. Shigesada, K. Kawasaki, and E. Teramoto, J. Theor. Biol. 79, 83 (1979).

[9] V. Capasso and A. Di Liddo, J. Math. Biol. 32, 453 (1994).

[10] Yu. A. Kuznetsov, M. Ya. Antonovsky, V. N. Biktashev, and E. A. Aponina, J. Math. Biol. 32, 219 (1994).

[11] J. Chattopadhyay, P. K. Tapaswi, D. Datta, and D. Chattopadhyay (Shakar), Ecol. Modell. 73, 205 (1994).

[12] B. Dubey, B. Das, and J. Hussain, Ecol. Modell. 141, 67 (2001).
[13] R. FitzHugh, Biophys. J. 1, 445 (1961); J. Nagumo, S. Arimoto, and S. Yoshizawa, Proc. IRE 50, 2061 (1962).

[14] S. M. Ali, A. Samanta, and S. K. Ghosh, J. Chem. Phys. 114, 10419 (2001); M. E. Schimpf and S. N. Semenov, Phys. Rev. E 70, 031202 (2004).

[15] D. del-Castillo-Negrete, B. A. Carreras, and V. Lynch, Physica D 168, 45 (2002).

[16] S. R. DeGroot and P. Mazur, Non-equilibrium Thermodynamics (Dover, New York, 1984); W. Ebeling and I. M. Sokolov, Statistical Thermodynamics and Stochastic Theory of Nonequilibrium Systems (World Scientific, Singapore, 2005).

[17] Piecewise linear approximations of nonlinear kinetic terms in reaction-diffusion equations have been widely used since about 1970, see H. P. McKean, Adv. Math. 4, 209 (1970). For an example in a population dynamics context, namely an invasion model with an Allee effect, see M.-H. Wang and M. Kot, Math. Biosci. 171, 83 (2001).

[18] J. Rinzel and J. B. Keller, Biophys. J. 13, 1313 (1973).

[19] V. Méndez and A. Compte, Physica A 260, 90 (1998).

[20] J. Rinzel and D. Terman, SIAM J. Appl. Math. 42, 1111 (1982).

[21] E. P. Zemskov, Phys. Rev. E 69, 036208 (2004). 